\title{
Immunomagnetic nanoparticle-based assays for detection of biomarkers
}

\author{
This article was published in the following Dove Press journal: \\ International Journal of Nanomedicine \\ 21 November 2013 \\ Number of times this article has been viewed
}

\author{
Hoyoung Park',2 \\ Mintai P Hwang' \\ Kwan Hyi Lee ${ }^{1,2}$ \\ 'Center for Biomaterials, Biomedical \\ Research Institute, Korea Institute of \\ Science and Technology, ${ }^{2}$ Department \\ of Biomedical Engineering, University \\ of Science and Technology, Seoul, \\ Republic of Korea
}

\begin{abstract}
The emergence of biomarkers as key players in the paradigm shift towards preventative medicine underscores the need for their detection and quantification. Advances made in the field of nanotechnology have played a crucial role in achieving these needs, and have contributed to recent advances in the field of medicine. Nanoparticle-based immunomagnetic assays, in particular, offer numerous advantages that utilize the unique physical properties of magnetic nanoparticles. In this review, we focus on recent developments and trends with regards to immunomagnetic assays used for detection of biomarkers. The various immunomagnetic assays are categorized into the following: particle-based multiplexing, signal control, microfluidics, microarray, and automation. Herein, we analyze each category and discuss their advantages and disadvantages.
\end{abstract}

Keywords: magnetic, multiplex, signal control, microfluidics, microarrays, automation

\section{Introduction}

The discovery of various biomarkers and the emergence of their clinical significance have given impetus to further changes in the constantly evolving field of health care. Specifically, biomarkers have become active players rather than mere catalysts in the paradigm shift from treatment-based medicine towards preventative medicine. With the latter placing an emphasis on the early detection and monitoring of diseases, biomarkers are being employed in a variety of methods and standards. In fact, according to the National Institutes of Health, biomarkers are "a characteristic that is objectively measured and evaluated as an indicator of normal biological processes, pathogenic processes, or pharmacologic responses to a therapeutic intervention". "The early detection of cancer and development of personalized detection and treatment modalities, for instance, could become possible through the use of such biomarkers, thereby underscoring their crucial role in the continuing development of medicine. Coupled with recent developments in the field of nanotechnology, biomarkers and their use in the detection of various diseases will only continue to grow.

Immunomagnetic assays, in particular, refer to identification of the target analyte (eg, antigen) via capture moieties (ie, antibodies, ligands, nucleotides) conjugated onto the surface of magnetic particles. Specifically, the capture moieties detect the desired target (ie, protein biomarker, DNA, RNA) for subsequent separation from the remaining solution via a simple magnet, and consequently, for various methods of analyses. $^{2-6}$ The noninvasiveness and simple nature of this method continues to enable the widespread use of magnetic particles. Furthermore, the high surface-area-to-volume ratio of the particles confers a correspondingly high probability of interaction with
Correspondence: Kwan Hyi Lee

Korea Institute of Science and

Technology, Hwarangno

|4-gil 5 Seongbuk-gu Seoul,

|36-79| Republic of Korea

Tel +8229586804

Fax +8229585308

Email kwanhyi@kist.re.kr 

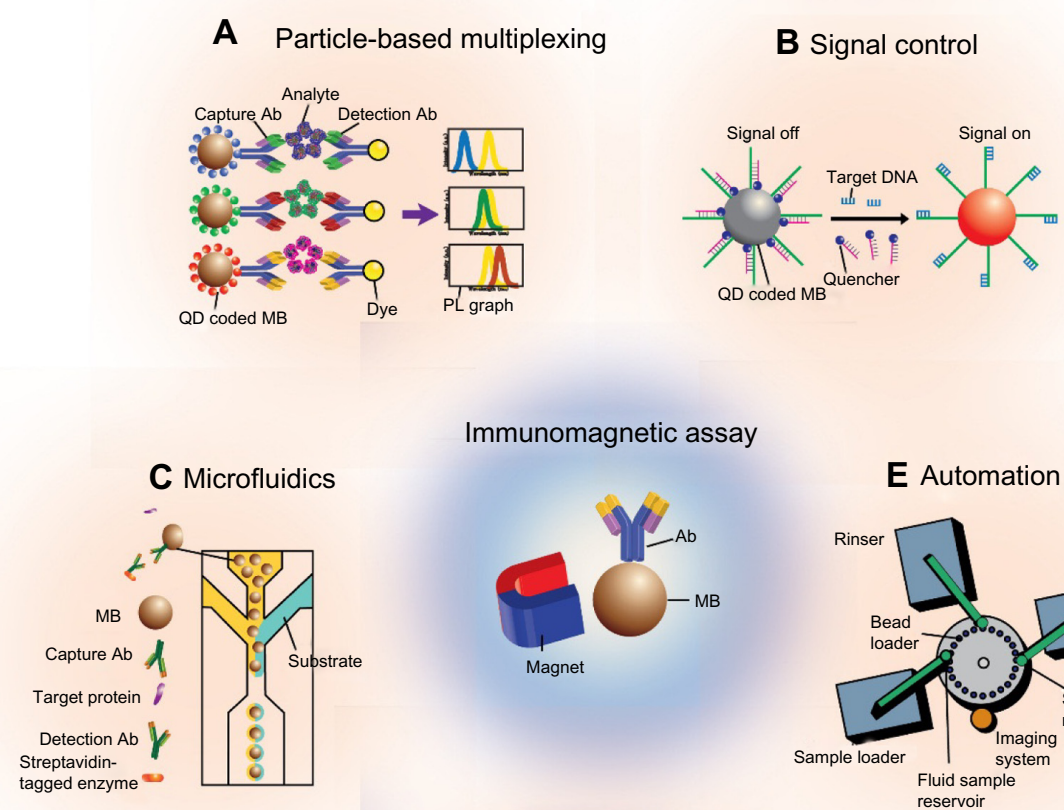

Immunomagnetic assay
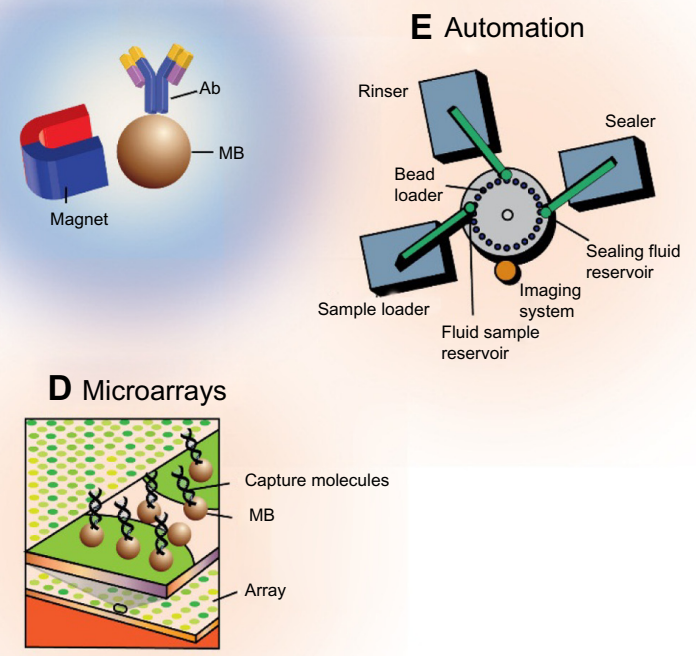

Figure I Schematic of current immunomagnetic assays.

Notes: (A and B) Particle-based assays and (C-E) device-based assays include microfluidics, microarrays, and automated immunomagnetic assays. Abbreviations: Ab, antibody; PL, photoluminescence; QD, quantum dot; MB, magnetic bead.

target biomarkers, and essentially increases the efficiency of the system. ${ }^{7}$ The stability of the particles in various chemical environments works to further increase their use across a wide range of applications. ${ }^{8}$ Additionally, the three-dimensionality of particles in solution can be used to increase the collection and separation efficiency of biomarkers; the use of conventional enzyme-linked immunosorbent assay (ELISA) kits, on the other hand, is limited by their two-dimensional approach to biomarker targeting. Finally, magnetic particles are often surface-functionalized and conjugated with other nanomaterials, including gold particles, quantum dots, and protein nanocages, to generate a platform for the highlysensitive quantification of biomarkers. ${ }^{9-11}$ The diagnosis of diseases should be time-efficient, accurate, and versatile. To reflect such needs, current developments within immunomagnetic assays include the simultaneous detection of multiple biomarkers and the use of micro/nanoscale devices to minimize sample volume. In this review, we categorize the use of immunomagnetic assays into particle-based multiplexing, signal control, microfluidic, microarray, and automated applications, and analyze their advantages and disadvantages (Figure 1).

\section{Particle-based multiplexing}

The ability to detect multiple biomarkers simultaneously offers an improved and more accurate approach to clinical diagnosis than that done with individual biomarkers in multiple steps (Figure 2A, B and C). Furthermore, such multiplexing methods are advantageous in their short processing time, minimization of sample volume, and economic cost. In particular, quantum dots are often used in the quantification and analysis of individual signals, which precludes the application of particle-based multiplexing methods for their unique optical properties, such as their narrow emission wavelength. ${ }^{12}$ Yet, the importance of maintaining the assay sensitivity, specificity, and reproducibility, in addition to the need for an encoding particle that converts the biomarker signal into a visible output, limit the widespread use of particle-based multiplex assays. 

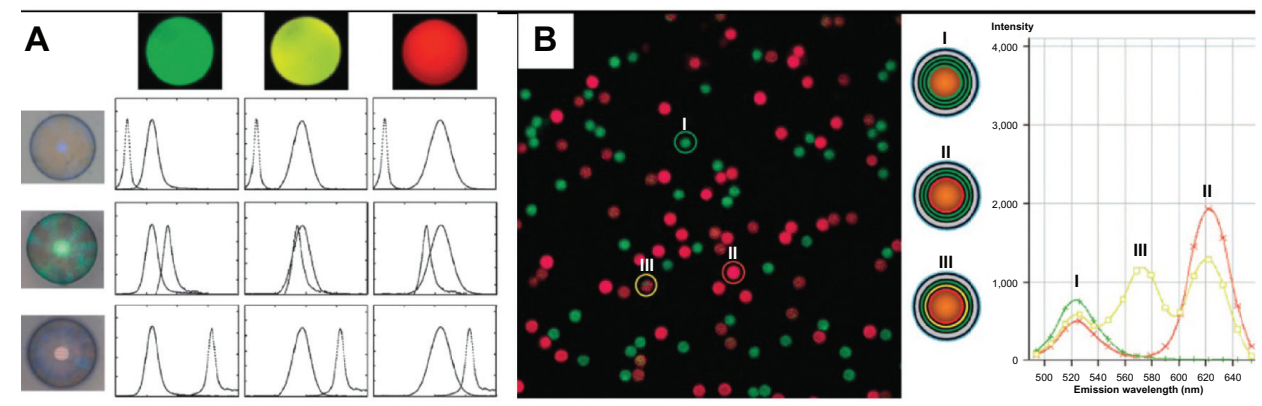

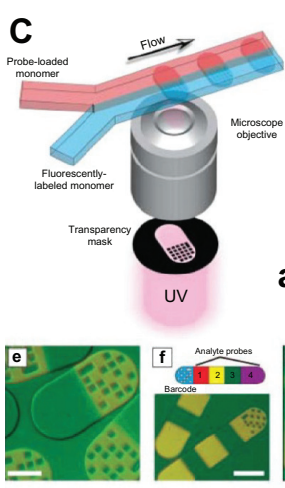

E

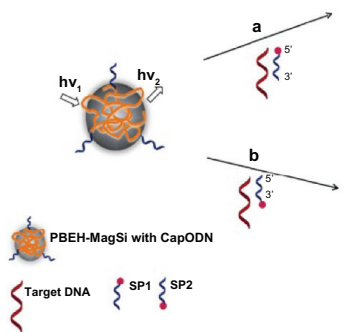

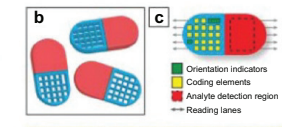

a
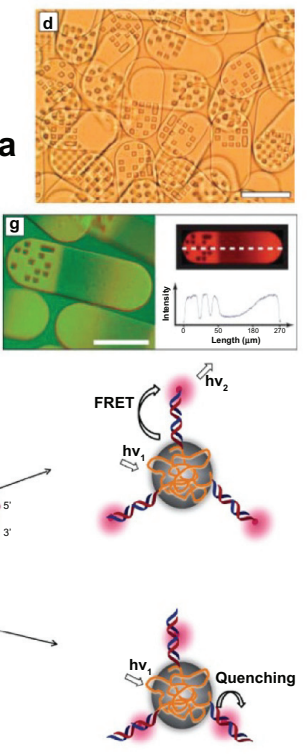

D
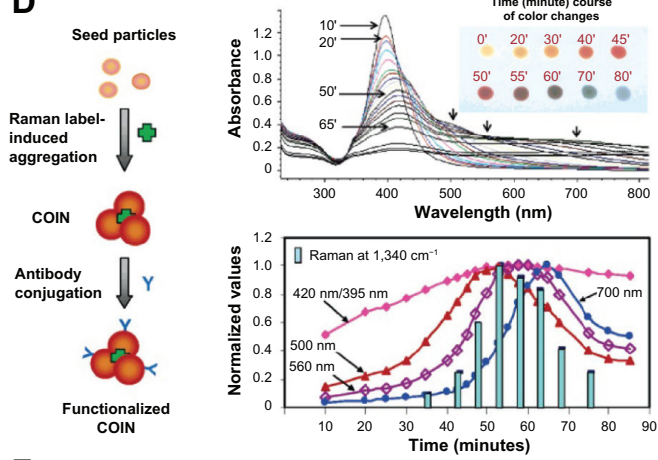

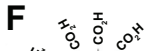

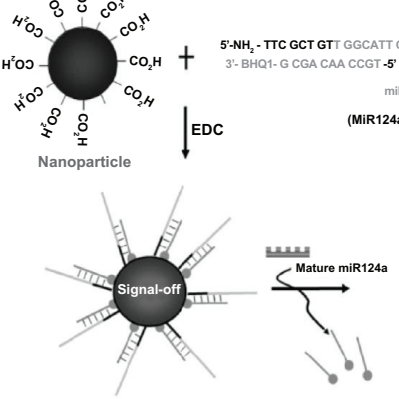
C CGCGTG CCTTAA - -3'

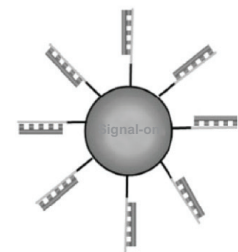

Figure 2 Examples of particle-based immunomagnetic assays.

Notes: (A) Various fluorescent dyes are coated onto the surface of magnetic particles for multiplexed detection of biomarker. ${ }^{61}$ (B) Different ratios of green, yellow, and red fluorescent dyes are layered onto magnetic particles for multiplexed detection of biomarker. ${ }^{60}$ (C) Barcode magnetic particles are used to consolidate information from multiple encoding particles. ${ }^{43}$ (a) Schematic diagram of dot-coded particle synthesis showing polymerization across two adjacent laminar streams to make single-probe, halffluorescent particles (shown in b); (c) diagrammatic representation of particle features for encoding and analyte detection. Encoding scheme shown allows the generation of $2^{20}(I, 048,576)$ unique codes; (d) differential interference contrast (DIC) image of particles generated by using the scheme shown in (a); (e-g) overlap of fluorescence and DIC images of single-probe (e), multiprobe (f, bottom), and probe-gradient ( $\mathbf{g}$, left) encoded particles. A schematic representation of multiprobe particles is also shown (f, top) and a plot of fluorescent intensity along the center line of a gradient particle (g, right). (D) COINs are fabricated for amplification of the Raman signal. ${ }^{18}$ (E and $\mathbf{F}$ ) Smart magnetic particles, such as those that release a quenching molecule upon biomarker recognition for subsequent fluorescence analysis, are fabricated. ${ }^{21,62}$ (E) Principles of DNA detection systems based on two different routes, namely FRET (a) and superquenching (b) PBEH modified magnetic beads (PBEH-MagSi) immobilized with CapODNs form sandwich assays with SPI/SP2. (A) Reproduced from Li J, Zhao XW, Zhao YJ, et al. Quantum-dot-coated encoded silica colloidal crystals beads for multiplex coding. Chem Commun (Camb). 2009;(I7):2329-233I with permission of The Royal Society of Chemistry. (B) Reprinted with permission from Wilson R, Spiller DG, Prior IA, et al. A simple method for preparing spectrally encoded magnetic beads for multiplexed detection. ACS Nano. 2007;I (5):487-493. Copyright 20I3 American Chemical Society. (C) From Pregibon DC, Toner M, Doyle PS. Multifunctional encoded particles for high-throughput biomolecule analysis. Science. 2007;315(58I7): 1393-1396. Reprinted with permission from AAAS. (D) Reprinted with permission from Su X, Zhang J, Sun L, et al. Composite organic-inorganic nanoparticles (COINs) with chemically encoded optical signatures. Nano Lett. 2005;5(I):49-54. Copyright 2013 American Chemical Society. (E) Reprinted from Biosensors and Bioelectronics, 35/I, Srinivas ARG, Peng H, Barker D, Travas-Sejdic J, Switch on or switch off: an optical DNA sensor based on poly(p-phenylenevinylene) grafted magnetic beads, 498-502, Copyright 2013, with permission from Elsevier. (F) Reprinted with permission from Hwang DW, Song IC, Lee DS, Kim S. Smart magnetic fluorescent nanoparticle imaging probes to monitor microRNAs. Small. 2010;6(I): 8I-88. Copyright @) 2013 Wiley-VCH Verlag GmbH \& Co. KGaA, Weinheim.

Abbreviations: COIN, composite organic-inorganic nanoparticles; UV, ultraviolet; FRET, fluorescence resonance energy transfer; PBEH, poly(2,5-bis[ethyl-7-heptanoate]p-phenylenevinylene-alt-phenylenevinylene); ODN, oligonucleotides; EDC, N-ethyl-NO-dimethylaminopropyl carbodiimide.

Martin et al utilized differing numbers of magnetic particles to distinguish among the samples. ${ }^{13} \mathrm{~A}$ set of magnetic particles specific for one biomarker is coated with one type of fluorescent molecule while another set specific for another biomarker is coated with a second type of fluorescent molecule. Biomarkers of interest are then sandwich-targeted with a detection antibody conjugated with a third type of fluorescent molecule. By varying the number of each type of magnetic particle as well as the type of biomarker present in solution, the authors were able to demonstrate simultaneous detection of multiple biomarkers. However, the need to guarantee nonoverlapping fluorescent emission spectra limits 


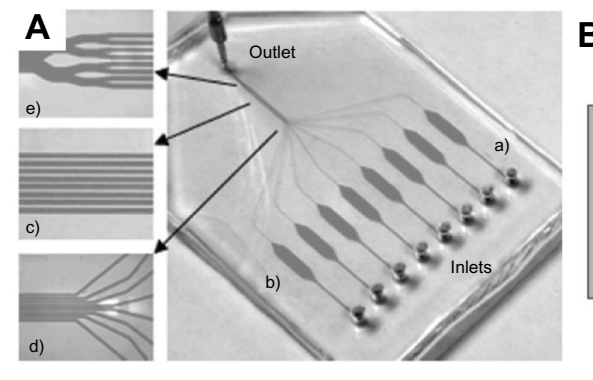

B
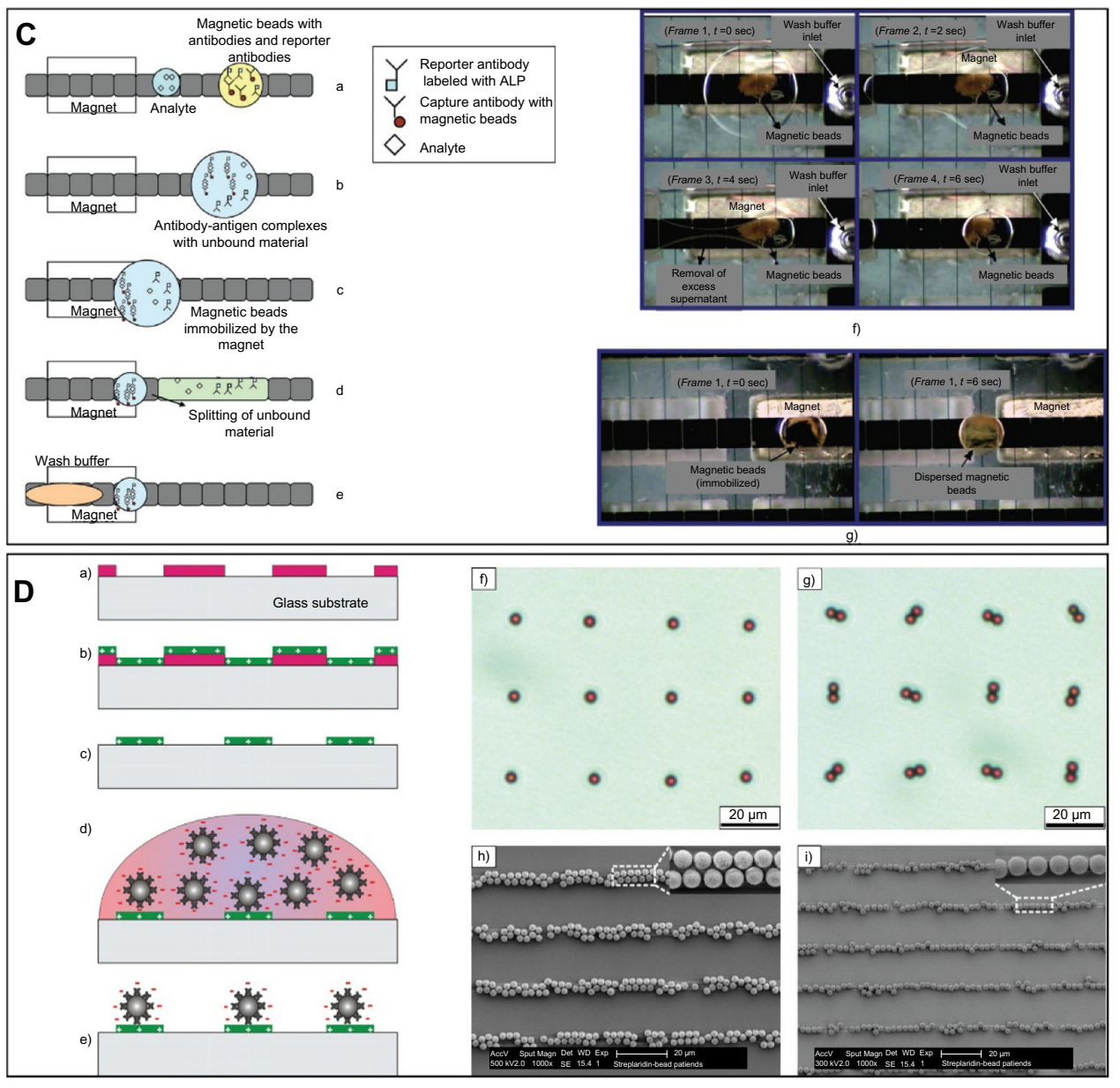

Figure 3 Microfluidics-based immunomagnetic devices for detection of biomarker.

Notes: (A) Magnetic particles react with samples in microfluidic chambers for simultaneous analysis of multiple samples ${ }^{40}$ PDMS chip for the realization of simultaneous microfluidic ELISA: a, individual channel with a cross-section of $200 \times 50 \mu \mathrm{m}$ acting as a diffusion barrier; b, reaction chamber with dimensions of $6 \mathrm{~mm} \times 2 \mathrm{~mm} \times 50 \mu \mathrm{m}(\mathrm{L} \times \mathrm{W} \times \mathrm{H})$; c, network of channels with a cross-section of $50 \times 50 \mu \mathrm{m}$ referred as the detection area; d, gathering of the independent channels; $\mathbf{e}$, merging of the channels into a unique outlet channel connected to a syringe pump in withdrawal mode. (B) Magnetic particles are incorporated into isolated droplets within microchannels for subsequent analysis in a stable microenvironment free from confounding factors. ${ }^{33}$ Photographs of the magnetic fusion of two droplets in the reaction chamber filled with mineral oil. The droplet containing superparamagnetic beads and $5 \mu \mathrm{l} \mathrm{NaOH}$ was manipulated toward a droplet containing phenol red. a, before fusion; $\mathbf{b}, \mathrm{t}=0 \mathrm{~s} ; \mathbf{c}, \mathrm{t}=30 \mathrm{~s} ; \mathbf{d}$, $\mathrm{t}=60 \mathrm{~s}$. (C) Magnetic particle loss is minimized via use of electrowetting. Removal of excess supernatant and washing of magnetic particles is done within droplets. ${ }^{38}$ Protocol for heterogeneous immunoassay on a digital microfluidics platform, a, dispensing of reagents; $\mathbf{b}$, incubation; $\mathbf{c}$, immobilization of magnetic beads; $\mathbf{d}$, removal of supernatant and washing; e, adding fresh wash buffer; $\mathbf{f}$, washing of magnetic beads by removing the excess supernatant on chip; $\mathbf{g}$, resuspension of magnetic beads. (D) Magnetic particles are self-assembled onto a glass substrate via electrostatic interactions for a highly reproducible and rapid ( $<30$ minutes) immunomagnetic asassy. ${ }^{44}$ Schematic illustration of the micropatterning process of streptavidincoated beads on a APTES template using electrostatic self-assembly. $\mathbf{a}$, positive photoresist micropattern on a glass substrate; $\mathbf{b}$, spin-coating of the APTES layer; $\mathbf{c}$, lift-off of the photoresist using ultrasonication; $\mathbf{d}$, a droplet of streptavidin-coated beads in PBS or PBST is incubated on the substrate; $\mathbf{e}$, pattern of self-assembled beads after rinsing; $\mathbf{f}$ and $\mathbf{g}$, optical micrographs showing streptavidin-coated beads $(\varnothing 2.8 \mu \mathrm{m})$ patterned in the form of singlet and doublets on the dot-arrays of dot size $2 \mu \mathrm{m} \mathrm{f}$, and $3 \mu \mathrm{m} \mathbf{g}$, respectively; $\mathbf{h}$ and $\mathbf{i}$, SEM micrographs showing streptavidin-coated beads $(\varnothing 2.8 \mu \mathrm{m})$ patterned in the form of stripe arrays of stripe width $3 \mu \mathrm{m} \mathbf{h}$, and $2 \mu \mathrm{m} \mathbf{i}$, respectively. (A) Reproduced from Herrmann M, Veres T, Tabrizian M. Enzymatically-generated fluorescent detection in micro-channels with internal magnetic mixing for the development of parallel microfluidic ELISA. Lab Chip. 2006;6(4): 555-560 with permission of The Royal Society of Chemistry. (B) Reprinted from Sensors and Actuators B: Chemical, I30/2, Tsuchiya H, Okochi M, Nagao N, et al, On-chip polymerase chain reaction microdevice employing a magnetic droplet-manipulation system, 583-588, Copyright 20I3, with permission from Elsevier. (C) Reproduced from Sista RS, Eckhardt AE, Srinivasan V, et al. Heterogeneous immunoassays using magnetic beads on a digital microfluidic platform. Lab Chip. 2008;8(I2):2188-2196 with permission of The Royal Society of Chemistry. (D) Reprinted from Sensors and Actuators B: Chemical, 132/2, Sivagnanam V, Sayah A, Vandevyver C, et al, Micropatterning of protein-functionalized magnetic beads on glass using electrostatic self-assembly, 36I-367, Copyright 2013, with permission from Elsevier.

Abbreviation: ALP, alkaline phosphatase; ELISA, enzyme-linked immunosorbent assay; PDMS, poly (dimethylsiloxane); APTES, (3-Aminopropyl)triethoxysilane; SEM, scanning electron microscope; PBS, phosphate buffered saline; PBST, phosphate buffered saline with tween. 


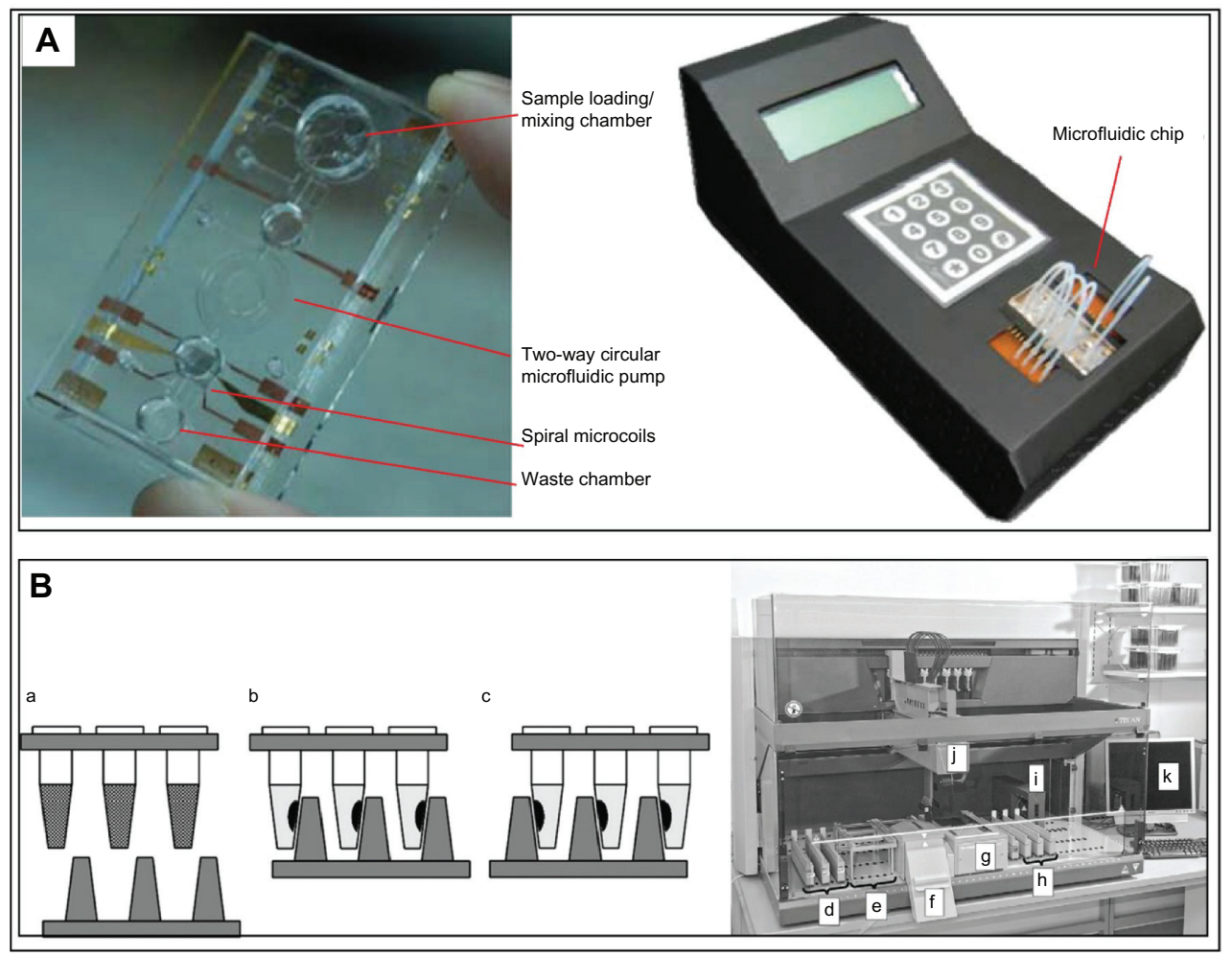

Figure 4 Automated immunomagnetic devices.

Notes: (A) A portable magnetic particle-based microfluidic device is used for detection of DNA biomarker. ${ }^{63}$ (B) Pillar-shaped magnets are used for automated transportation, washing, and detection of DNA biomarkers. ${ }^{51}$ Working principle of the magnetic separator (B); $\mathbf{a}$, metal plate with the reaction tubes containing magnetic particles suspended in buffer solution; $\mathbf{b}$, and $\mathbf{c}$, for the washing steps, the metal plate with the magnets can be moved from one side of the tube to the other; $\mathbf{d}$, carriers for sample lysates; $\mathbf{e}$, carriers for disposable tips; f, waste container; g, magnetic separator; h, carriers for DNA eluates; i, barcode reader, j, liquid handling arm and $\mathbf{k}$, computer. (A) Reprinted from Microfluidics and Nanofluidics, 6/4, Lien KY, Liu CJ, Lin YC, et al, Extraction of genomic DNA and detection of single nucleotide polymorphism genotyping utilizing an integrated magnetic bead-based microfluidic platform, 539-555, Copyright 2013, with kind permission from Springer Science and Business Media. (B) Reprinted from Forensic Science International: Genetics, 6/5, Witt S, Neumann J, Zierdt H, et al, Establishing a novel automated magnetic bead-based method for the extraction of DNA from a variety of forensic samples, 539-547, Copyright 2013, with permission from Elsevier.

the number of biomarkers that can be detected simultaneously and increases the number of encoding particles needed.

To improve on these limitations, Bong et al ${ }^{14}$ used barcode magnetic beads to incorporate multiple encoding particles into a single particle. The transmission of light, or the presence/absence of fluorescence upon light exposure onto the particles that have been integrated with unique patterns in the form of a barcode, provide information that the authors decode to demonstrate the multiplexed detection of biomarkers. ${ }^{14}$ Similarly, others have utilized magnetic beads coated with a variety of fluorescent dyes to demonstrate the ability to simultaneously detect various cytokines (ie, interleukin-8 and interleukin-2) and cancer biomarkers (ie, epidermal growth factor, sCD40L, and Apolipoprotein A-I). ${ }^{15,16}$ Further improvements are made to the system through conjugation of various DNA probes onto the barcode. Different probes/primers labeled using a dye with a unique emission spectrum are used in conjunction with the magnetic particles to increase the multiplexity of biomarker detection. ${ }^{17}$

In addition to the use of fluorescent signals, surfaceenhanced Raman signals have been used in the detection of biomarkers. Specifically, Raman signals utilize the different molecular vibrations of scattered light, which possess unique optical properties compared with incident light. In particular, Su et al, and Sun et al used composite organic-inorganic nanoparticles for the multiplexed detection of biomarkers. Specifically, by varying the ratio of organic to inorganic materials, the authors fabricated varying composite organic-inorganic nanoparticle interior structures and Raman-active compounds. ${ }^{18,19}$ Such detection methods alleviate issues of broad emission spectra and photobleaching associated with fluorescent dyes. Furthermore, large expensive devices commonly used in the detection of fluorescent signals are not needed in detection of surface-enhanced Raman signals, thereby opening up avenues for miniaturized devices. However, surface-enhanced Raman signal-based assays are limited by their relatively weak Raman signal, thus requiring amplification of the signal while maintaining an optimal signal-to-noise ratio. ${ }^{20}$

\section{Signal control}

The widespread use of biomarker multiplexing is precluded by the need to reduce background noise while specifically 
detecting the desired target. This condition is particularly important when considering the conglomeration of nontarget biomarkers present in ex vivo samples such as blood or urine. Therefore, the ability to control the output signal is a vital factor in accurate detection of the desired biomarker (Figure 2D, E and F).

Hwang et al utilized rhodamine-coated cobalt ferrite magnetic fluorescent beads for detection of the desired biomarker. After conjugating black hole-quenching molecules onto the magnetic fluorescent beads, which act to quench the fluorescence, the authors demonstrated the ability to selectively detach black hole-quenching molecules upon binding of target biomarker. In essence, the authors controlled the fluorescent signal to detect the desired biomarker by analyzing the fluorescence after black hole-quenching molecule detachment. ${ }^{21}$

Immunomagnetic assays that amplify the output signal are used as well, particularly when the target biomarker exists in minute amounts. The augmentation of signal is generally accomplished via the coating of multiple nanosized particles (ie, quantum dots) onto microsized magnetic particles. Qian et al, for instance, demonstrated amplification of signal by coating quantum dots onto the surface of silica nanospheres. ${ }^{22}$ Similarly, Li et al formed ultrathin silica or alumina shells around gold nanoparticles and demonstrated amplification of Raman signal for various molecules adsorbed onto single-crystal surfaces. ${ }^{23}$ Taking a slightly different approach, Liu et al used silver nanoparticles to target DNA, which were subsequently dissolved to yield a large amount of silver ions for chemiluminescent signal amplification. ${ }^{24}$ Amplified electrochemical signals derived from magnetic nanospheres coated with multiple gold particles have also been used for the detection of myoglobin and carcinoembryonic antigen. Specifically, horseradish peroxidase-conjugated secondary antibodies were used with gold-functionalized magnetic nanospheres to sandwich-target the biomarkers, upon which the reaction of horseradish peroxidase further amplifies the electrochemical signal..$^{25,26}$

The immunomagnetic assays described thus far present advantages over those that utilize microarrays or microfluidic devices, in that the magnetic particles move freely within the sample solution to maximize binding to target biomarkers. Furthermore, the assays are simple to use and provide a high-throughput solution for the detection of multiple biomarkers. On the other hand, the need for multiple washing steps, the relatively high cost due to use of millions of magnetic particles and a correspondingly large amount of reagents, and the lengthy analysis time remain undesirable factors in the use of such assays.

For practical use in the field and clinic, an assay that reflects the need for rapid detection and analysis of a biomarker is needed. In addition, factors such as the sensitivity of the assay and the amount of reagents used should be taken into consideration as an economical means to detect minute amounts of biomarker. Device-based immunomagnetic assays (ie, microfluidics, microarrays) have been conceived to accommodate such needs and remedy the aforementioned shortcomings.

\section{Microfluidics}

Conventional means of microfluidic immunomagnetic assays involve the use of a simple magnet to separate and collect biomarker-bound magnetic particles from within a microfluidic channel containing both biomarker and magnetic particles conjugated with capture moieties (Figure 3). ${ }^{27-29}$ For accurate detection of biomarker, the test solution must be well mixed within the microfluidic channel. Accordingly, enhanced microfluidic immunomagnetic assays have been developed to address the issue of laminar flow-induced non-mixing, a commonly observed phenomenon in conventional microfluidic assays.

For instance, Sasso et al demonstrated the ability to detect tumor necrosis factor- $\alpha$ and interleukin- 6 by placing a magnet at the junction of two inlets for biomarker solution and functionalized magnetic particles. Here, the magnet works to ensure thorough mixing of the two through application of a magnetic force perpendicular to the flow of the test solution. ${ }^{30,31}$ Kim et al further improved the assay by retaining the same inlet system and modifying the outlets to include a magnet for separation and purification of the targeted biomarker in one outlet and the remaining solution of nontarget protein in another. ${ }^{32}$

Apart from ensuring even mixing, microfluidic assays should also be able to preserve the detection microenvironment. To meet these needs, microfluidic immunomagnetic assays that use droplets to encapsulate both functionalized magnetic particles and reagents in a single microenvironment have been proposed..$^{33-37}$ Such droplet microfluidic assays are advantageous in their capacity to maintain a stable microenvironment within the droplet, minimize surface adsorption of target biomarker onto the channel walls, and prevent diffusioninduced spreading of contaminants and fluctuations in reagent concentration. Yet, the lack of reconfigurability, due to the channel-based continuous flow format, and issues of channel clogging remain as obstacles. Hence, the ability to control and handle the droplets is needed. Sista et al, for instance, detected interleukin-6 within droplets, followed by removal of excess supernatant, washing of magnetic particles, and resuspension of particles within each droplet. ${ }^{38}$

Magnetic particles are also being used together with microfluidic channels. For instance, magnetic particles are used as a solid support through their immobilization onto 
the microfluidic channels. ${ }^{39}$ The increased surface-to-volume ratio and therefore amplified sensitivity, coupled with high reproducibility, present further advantages that have been utilized by others. ${ }^{39-42}$ In another example, barcoded particles segmented into two parts for detection of the biomarker and fluorescent signals, are used in conjunction with two microfluidic channels through which the sample flows. ${ }^{43}$ Such methods utilize minimal amounts of reagent and are capable of multiplexed biomarker detection.

\section{Microarrays}

Protein microarrays are often used for screening applications, in which a large number of potential targets need to be analyzed simultaneously. Despite this advantage, conventional protein microarrays are hardly time and cost-efficient due to their large surface area. Furthermore, the addition or removal of a spot is difficult. For example, an increase in the number of spots from 100 to 101 requires respotting the entire device. The combination of magnetic particles and microarrays has thus been proposed to address such shortcomings.

Sivagnanam et al developed a method to uniformly adsorb functionalized magnetic particles onto the surface of a glass substrate via electrostatic interactions. ${ }^{44}$ By performing a microarray analysis of the system, the authors demonstrated an enhanced specific binding surface. Separately, Kan et al, and Rissin et al used a microarray system in which biomarkerbound magnetic particles are placed into each microwell for quantitative analysis. ${ }^{45,46} \mathrm{Such}$ methods provide a means to overcome the limitations of conventional microarrays, particularly with regards to the inflexibility of the system. Similarly, Bergo placed biomarker-bound magnetic particles into microwells, but also eluted the bound biomarkers to form discrete spots for microarray analysis via mass spectrometry. ${ }^{47}$ This method presents an advantage over conventional use of magnetic particles with microarrays in that the biomarker of interest is separated from magnetic particles for more accurate measurement and analysis.

\section{Automation}

Detection of biomarkers in the field ideally requires minimal labor via an automated system. The combination of this condition with the distinct advantage conferred by immunomagnetic assays (ie, easy separation of biomarker-bound particles via a simple magnet) has resulted in the continued development of time-efficient and automated immunomagnetic assays (Figure 4). For instance, Chen CL et al, and Chen $\mathrm{KC}$ et al used a disk-shaped carrier board to fabricate a microchannel device into which biomarkers, magnetic particles, and antibodies conjugated with an imaging dye could be automatically and sequentially injected for analysis. ${ }^{48-50}$ In addition to such disk magnets, pillar-shaped magnets and microfluidic devices that use micropumps are also being used to transport, wash, and detect desired biomarkers (ie, DNA, C-reactive protein). ${ }^{51,52}$ The general direction of such automated devices is towards the assembly of a portable device. Along these lines, Son and Yoon combined quantum dot-coated magnetic particles with a microreactor chip to detect biomarkers of interest. ${ }^{53}$ In another example, a device that measures the magnetoresistance of magnetic particles upon targeting of the desired biomarker has also been used to demonstrate the feasibility of portable devices. ${ }^{54}$ Finally, Safar et al engineered a portable device with user control over the sample temperature. ${ }^{55}$ Ultimately, these research trends aim to utilize the numerous advantages conferred by immunomagnetic assays for the highly sensitive and specific multiplexed detection of biomarkers.

\section{Current and future developments}

Recent immunomagnetic assays can largely be categorized into particle-based and device-based assays. While particle-based assays are simple to use and represent a high-throughput method, they often require relatively large volumes of sample and reagent. On the other hand, device-based assays require only a small amount of sample and offer a rapid assay method, yet suffer from irregular mixing of the sample within the device. Consequently, assays that combine advantages from both types have emerged and are currently used for detection of biomarkers.

Furthermore, while individual components of immunomagnetic assays (eg, magnetic particles, microfluidic devices, surface-enhanced Raman signals, and quantum dots) have been widely developed, their combination has only recently started to generate interest. Here, we briefly include future directions and improvements that could be made to current immunomagnetic assays:

1. ELISA, one of the most traditionally used methods in the detection of various biomarkers, is a two-dimensional antigen targeting system from the perspective that the bottom of each well is coated with a capture antibody. The consequent importance in ensuring that the sample solution comes in contact with the surface alone limits the availability of target analyte binding to the antibodies. Coupled with the need for relatively large volumes of sample, ELISAs tend to be confined by their lower sensitive limit of detection values. From this perspective, the use of magnetic nanoparticles in solution confers a three-dimensional antigen-targeting environment, as well as a means to improve the limit of detection.

2. The relatively low sensitivity of ELISA has been alleviated by use of magnetic beads and various devices. 
For example, the limit of detection of prostate-specific antigen biomarker has been improved from $200 \mathrm{pg} / \mathrm{mL}$ (linear range $1.5-25 \mathrm{ng} / \mathrm{mL}$ ) as assessed by ELISA to $3 \mathrm{pg} / \mathrm{mL}$ (linear range $1-40 \mathrm{ng} / \mathrm{mL}$ ) by using a carbon nanotube-based magnetic bead system. ${ }^{56}$ Additionally, some have used magnetic beads in conjunction with quantum dots to yield an electrochemical-based limit of detection of $20 \mathrm{pg} / \mathrm{mL}$ (linear range $0.05-4 \mathrm{ng} / \mathrm{mL}$ ), ${ }^{57}$ while others have used magnetic grapheme with quantum dot-functionalized silica particles to obtain a limit of detection of $0.72 \mathrm{pg} / \mathrm{mL}$ (linear range $0.003-50 \mathrm{ng} / \mathrm{mL}$ ). ${ }^{58}$ As such, magnetic bead-based assays demonstrate improved sensitivity over enzyme-based ELISAs, and can be further improved by combining magnetic beads with other systems such as magnetic grapheme, silica, and quantum dot nanoparticles.

3. A combination of magnetic beads with various devices has also been shown to improve the sensitivity of biomarker detection. For instance, the limit of detection of C-reactive protein, which is approximately $1,000 \mathrm{ng} / \mathrm{mL}$ when detected by ELISA, can be improved to $130 \mathrm{ng} / \mathrm{mL}$ when magnetic beads and fluorescent dyes are used together in a microarray. Furthermore, a chemiluminescence-based assay that utilizes magnetic beads and microfluidic channels has demonstrated a limit of detection of $12.5 \mathrm{ng} / \mathrm{mL} .^{52}$

4. Use of barcode particles in a microfluidic device combines the capacity of barcode particles to retain multiple parameters of information (thereby reducing the number of necessary encoding particles) with the ability to use small sample volumes. Additional work in the form of the ability to extract barcode particles from the microfluidic device to obtain a more accurate signal is needed to further advance this assay.

5. Assays that provide control over an output signal for specific detection of a biomarker are currently limited to detection of DNA. The expanded ability to detect protein biomarkers would provide broader scope for these types of immunomagnetic assays.

6. Fluorescent signals are widely used and often amplified in the detection of target biomarkers. However, it is this amplification of fluorescent signal that often interferes with the input signal, leading to mixed results. From this perspective, the use of a nonfluorescent signal ${ }^{18,59}$ or the combination of a fluorescence signal with a nonfluorescent signal (ie, a surface-enhanced Raman signal) is a potential avenue for further development.

Continued development in other fields (ie, chemical, material, environmental) will undoubtedly result in novel targets of interest and a means to detect and quantify such biomarkers. Coupled with such developments, we envisage increased interest in immunomagnetic assays and a sustained and concerted effort towards their improvement.

\section{Acknowledgment}

The authors gratefully acknowledge the financial support provided by the Korea Institute of Science and Technology (Institutional Project \#2E23720 and Translational Research Center \#2E24203).

\section{Disclosure}

The authors report no conflicts of interest in this work.

\section{References}

1. Atkinson AJ, Colburn WA, DeGruttola VG, et al. Biomarkers and surrogate endpoints: preferred definitions and conceptual framework. Clin Pharmacol Ther. 2001;69(3):89-95.

2. Liandris E, Gazouli M, Andreadou M, Sechi LA, Rosu V, Ikonomopoulos J. Detection of pathogenic mycobacteria based on functionalized quantum dots coupled with immunomagnetic separation. PLoS One. 2011;6(5):e20026.

3. Yu LSL, Uknalis J, Tu SI. Immunomagnetic separation methods for the isolation of Campylobacter jejuni from ground poultry meats. J Immunol Methods. 2001;256(1-2):11-18.

4. Keating CD. Nanoscience enables ultrasensitive detection of Alzheimer's biomarker. Proc Natl Acad Sci U S A. 2005;102(7):2263-2264.

5. Botto L, Masserini M, Cassetti A, Palestini P. Immuno separation of Prion protein-enriched domains from other detergent-resistant membrane fractions, isolated from neuronal cells. FEBS Lett. 2004;557(1-3): 143-147.

6. Song LA, Shan DD, Zhao MW, et al. Direct detection of bacterial genomic DNA at sub-femtomolar concentrations using single molecule arrays. Anal Chem. 2013;85(3):1932-1939.

7. Sun Y, Bai YP, Song DQ, Li XZ, Wang U, Zhang HQ. Design and performances of immunoassay based on SPR biosensor with magnetic microbeads. Biosens Bioelectron. 2007;23(4):473-478.

8. Rife JC, Miller MM, Sheehan PE, Tamanaha CR, Tondra M, Whitman LJ. Design and performance of GMR sensors for the detection of magnetic microbeads in biosensors. Sens Actuators A Phys. 2003;107(3): 209-218.

9. Liu GD, Lin YH. Nanomaterial labels in electrochemical immunosensors and immunoassays. Talanta. 2007;74(3):308-317.

10. Tennico YH, Hutanu D, Koesdjojo MT, Bartel CM, Remcho VT. Onchip aptamer-based sandwich assay for thrombin detection employing magnetic beads and quantum dots. Anal Chem. 2010;82(13): $5591-5597$.

11. Wang J, Liu GD, Wu H, Lin YH. Quantum-dot-based electrochemical immunoassay for high-throughput screening of the prostate-specific antigen. Small. 2008;4(1):82-86.

12. Su XL, LiYB. Quantum dot biolabeling coupled with immunomagnetic separation for detection of Escherichia coli O157: H7. Anal Chem. 2004;76(16):4806-4810.

13. Martin J, Nguyen QN, inventors. Highly multiplexed particle based assays. United States Patent US 20120202293. May 5, 2012.

14. Bong KW, Chapin SC, Doyle PS. Magnetic barcoded hydrogel microparticles for multiplexed detection. Langmuir. 2010;26(11):8008-8014.

15. Jayadev C, Rout R, Price A, Hulley P, Mahoney D. Hyaluronidase treatment of synovial fluid to improve assay precision for biomarker research using multiplex immunoassay platforms. J Immunol Methods. 2012;386(1-2):22-30.

16. Kim BK, Lee JW, Park PJ, et al. The multiplex bead array approach to identifying serum biomarkers associated with breast cancer. Breast Cancer Res. 2009;11(2):R22. 
17. Chen G, Ho WZ, inventors. Highly multiplexed real-time PCR using encoded microbeads. United States Patent US20120088691. 2012 April 12

18. Su X, Zhang J, Sun L, et al. Composite organic-inorganic nanoparticles (COINs) with chemically encoded optical signatures. Nano Lett 2005;5(1):49-54.

19. Sun L, Sung KB, Dentinger C, et al. Composite organic-inorganic nanoparticles as Raman labels for tissue analysis. Nano Lett. 2007;7(2) 351-356.

20. Jun BH, Kim JH, Park H, et al. Surface-enhanced Raman spectroscopic-encoded beads for multiplex immunoassay. J Comb Chem. 2007;9(2):237-244.

21. Hwang DW, Song IC, Lee DS, Kim S. Smart magnetic fluorescent nanoparticle imaging probes to monitor microRNAs. Small. 2010;6(1): 81-88.

22. Qian J, Zhang CY, Cao XD, Liu SQ. Versatile immunosensor using a quantum dot coated silica nanosphere as a label for signal amplification. Anal Chem. 2010;82(15):6422-6429.

23. Li JF, Huang YF, Ding Y, et al. Shell-isolated nanoparticle-enhanced Raman spectroscopy. Nature. 2010;464(7287):392-395.

24. Liu CH, Li ZP, Du BA, Duan XR, Wang YC. Silver nanoparticlebased ultrasensitive chemiluminescent detection of DNA hybridization and single-nucleotide polymorphisms. Anal Chem. 2006;78(11): 3738-3744.

25. Gan N, Wang LH, Li TH, Sang WG, Hu FT, Cao YT. A novel signalamplified immunoassay for myoglobin using magnetic core-shell Fe3O4@Au-multi walled carbon nanotubes composites as labels based on one piezoelectric sensor. Integr Ferroelectr. 2013;144(1):29-40.

26. Tang DP, Yuan R, Chal YQ. Ultrasensitive electrochemical immunosensor for clinical immunoassay using thionine-doped magnetic gold nanospheres as labels and horseradish peroxidase as enhancer. Anal Chem. 2008;80(5):1582-1588.

27. Gijs MAM. Magnetic bead handling on-chip: new opportunities for analytical applications. Microfluid Nanofluidics. 2004;1(1):22-40

28. Zhang RQ, Liu SL, Zhao W, et al. A simple point-of-care microfluidic immunomagnetic fluorescence assay for pathogens. Anal Chem 2013;85(5):2645-2651.

29. Sasso LA, Undar A, Zahn JD. Autonomous magnetically actuated continuous flow microimmunofluorocytometry assay. Microfluid Nanofluidics. 2010;9(2-3):253-265

30. Sasso LA, Johnston IH, Zheng M, Gupte RK, Undar A, Zahn JD Automated microfluidic processing platform for multiplexed magnetic bead immunoassays. Microfluid Nanofluidics. 2012;13(4):603-612.

31. Sasso LA, Aran K, Guan YL, Undar A, Zahn JD. Continuous monitoring of inflammation biomarkers during simulated cardiopulmonary bypass using a microfluidic immunoassay device - a pilot study. Artif Organs. 2013;37(1):E9-E17.

32. Kim J, Lee HH, Steinfeld U, Seidel H. Fast capturing on micromagnetic cell sorter. IEEE Sens J. 2009;9(8):908-913.

33. Tsuchiya H, Okochi M, Nagao N, Shikida M, Honda H. On-chip polymerase chain reaction microdevice employing. a magnetic dropletmanipulation system. Sens Actuators B Chem. 2008;130(2):583-588.

34. Bronzeau S, Pamme N. Simultaneous bioassays in a microfluidic channel on plugs of different magnetic particles. Anal Chim Acta. 2008;609(1):105-112.

35. Boles DJ, Benton JL, Siew GJ, et al. Droplet-based pyrosequencing using digital microfluidics. Anal Chem. 2011;83(22):8439-8447.

36. Yoon S, Kim JA, Lee SH, Kim M, Park TH. Droplet-based microfluidic system to form and separate multicellular spheroids using magnetic nanoparticles. Lab Chip. 2013;13(8):1522-1528.

37. Witters D, Knez K, Ceyssens F, Puers R, Lammertyn J. Digital microfluidics-enabled single-molecule detection by printing and sealing single magnetic beads in femtoliter droplets. Lab Chip. 2013;13(11): 2047-2054.

38. Sista RS, Eckhardt AE, Srinivasan V, Pollack MG, Palanki S, Pamula VK. Heterogeneous immunoassays using magnetic beads on a digital microfluidic platform. Lab Chip. 2008;8(12):2188-2196.

39. Lim CT, Zhang Y. Bead-based microfluidic immunoassays: the next generation. Biosens Bioelectron. 2007;22(7):1197-1204.
40. Herrmann M, Veres T, Tabrizian M. Enzymatically-generated fluorescent detection in micro-channels with internal magnetic mixing for the development of parallel microfluidic ELISA. Lab Chip. 2006;6(4): 555-560.

41. Yu X, Xia HS, Sun ZD, et al. On-chip dual detection of cancer biomarkers directly in serum based on self-assembled magnetic bead patterns and quantum dots. Biosens Bioelectron. 2013;41:129-136.

42. Ren L, Wang JC, Liu WM, et al. An enzymatic immunoassay microfluidics integrated with membrane valves for microsphere retention and reagent mixing. Biosens Bioelectron. 2012;35(1):147-154.

43. Pregibon DC, Toner M, Doyle PS. Multifunctional encoded particles for highthroughput biomolecule analysis. Science. 2007;315(5817): 1393-1396.

44. Sivagnanam V, Sayah A, Vandevyver C, Gijs MAM. Micropatterning of protein-functionalized magnetic beads on glass using electrostatic self-assembly. Sens Actuators B Chem. 2008;132(2):361-367.

45. Kan CW, Rivnak AJ, Campbell TG, et al. Isolation and detection of single molecules on paramagnetic beads using sequential fluid flows in microfabricated polymer array assemblies. Lab Chip. 2012;12(5): 977-985.

46. Rissin DM, Kan CW, Song L, et al. Multiplexed single molecule immunoassays. Lab Chip. 2013;13(15):2902-2911.

47. Bergo VB, inventor. Devices and methods for producing and analyzing microarrays. United States Patent US20120202709. 2012 August 9.

48. Chen CL, Chen KC, Pan YC, et al. Separation and detection of rare cells in a microfluidic disk via negative selection. Lab Chip. 2011;11(3):474-483.

49. Chen KC, Pan YC, Chen CL, Lin CH, Huang CS, Wo AM. Enumeration and viability of rare cells in a microfluidic disk via positive selection approach. Anal Biochem. 2012;429(2):116-123.

50. Chen KC, Lee TP, Pan YC, et al. Detection of circulating endothelial cells via a microfluidic disk. Clin Chem. 2011;57(4):586-592.

51. Witt S, Neumann J, Zierdt H, Gebel G, Roscheisen C. Establishing a novel automated magnetic bead-based method for the extraction of DNA from a variety of forensic samples. Forensic Sci Int Genet. 2012;6(5): 539-547.

52. Lee WB, Chen YH, Lin HI, Shiesh SC, Lee GB. An integrated microfluidic system for fast, automatic detection of C-reactive protein. Sens Actuators B Chem. 2011;157(2):710-721.

53. Son A, Yoon Y, inventors. Magnetic bead quantum dot nanoparticle assay. United States Patent US20130029333. 2013 January 31.

54. Gaster RS, Hall DA, Wang SX. nanoLAB: an ultraportable, handheld diagnostic laboratory for global health. Lab Chip. 2011;11(5): 950-956.

55. Safar SG, Galitz CM, Herchenbach SL, Dunn CM, McDowell LL, inventors. Structure and method for handling magnetic particles in biological assays. United States patent US20120269702. 2012 October 25.

56. Chikkaveeraiah BV, Bhirde A, Malhotra R, Patel V, Gutkind JS, Rusling JF. Single-wall carbon nanotube forest arrays for immunoelectrochemical measurement of four protein biomarkers for prostate cancer. Anal Chem. 2009;81(21):9129-9134.

57. Liu GD, Lin YY, Wang J, Wu H, Wai CM, Lin YH. Disposable electrochemical immunosensor diagnosis device based on nanoparticle probe and immunochromatographic strip. Anal Chem. 2007;79(20): 7644-7653.

58. Liu F, Zhang Y, Ge SG, et al. Magnetic graphene nanosheets based electrochemiluminescence immunoassay of cancer biomarker using CdTe quantum dots coated silica nanospheres as labels. Talanta. 2012;99:512-519.

59. Huh YS, Lowe AJ, Strickland AD, Batt CA, Erickson D. Surfaceenhanced raman scattering based ligase detection reaction. J Am Chem Soc. 2009;131(6):2208-2213.

60. Wilson R, Spiller DG, Prior IA, Veltkamp KJ, Hutchinson A. A simple method for preparing spectrally encoded magnetic beads for multiplexed detection. ACS Nano. 2007;1(5):487-493.

61. Li J, Zhao XW, Zhao YJ, Gu ZZ. Quantum-dot-coated encoded silica colloidal crystals beads for multiplex coding. Chem Commun (Camb). 2009;(17):2329-2331.

62. Srinivas ARG, Peng H, Barker D, Travas-Sejdic J. Switch on or switch off: an optical DNA sensor based on poly(p-phenylenevinylene) grafted magnetic beads. Biosens Bioelectron. 2012;35(1):498-502.

63. Lien KY, Liu CJ, Lin YC, Kuo PL, Lee GB. Extraction of genomic DNA and detection of single nucleotide polymorphism genotyping utilizing an integrated magnetic bead-based microfluidic platform. Microfluid Nanofluidics. 2009;6(4):539-555. 


\section{Publish your work in this journal}

The International Journal of Nanomedicine is an international, peerreviewed journal focusing on the application of nanotechnology in diagnostics, therapeutics, and drug delivery systems throughout the biomedical field. This journal is indexed on PubMed Central, MedLine, CAS, SciSearch $\AA$, Current Contents ${ }^{\circledR} /$ Clinical Medicine,
Journal Citation Reports/Science Edition, EMBase, Scopus and the Elsevier Bibliographic databases. The manuscript management system is completely online and includes a very quick and fair peer-review system, which is all easy to use. Visit http://www.dovepress.com/ testimonials.php to read real quotes from published authors.

Submit your manuscript here: http://www.dovepress.com/international-journal-of-nanomedicine-journal 